10 years ESJ

Special edition

\title{
The Centrality of Language in Health Communication
}

Franca Daniele, $M D$

Department of Medical, Oral and Biotechnological Sciences

"G. d'Annunzio" University, Chieti-Pescara, Italy

Doi: 10.19044/esj.2021.v17n30p24

Submitted: 21 June 2021

Accepted: 15 July 2021

Published: 10 September 2021
Copyright 2021 Author(s)

Under Creative Commons BY-NC-ND

4.0 OPEN ACCESS

Cite As:

Daniele F. (2021). The Centrality of Language in Health Communication. European Scientific Journal, ESJ, 17 (30), 24. https://doi.org/10.19044/esj.2021.v17n30p24

\begin{abstract}
Medical communication and health communication are two close relatives in the field of communication, where medical communication is the mother and health communication is the offspring. Medical communication engages the delivery of scientific, medical, pharmaceutical and biotechnological information and data to health professionals like doctors, pharmacists, nurses, etc. The information includes updates on the latest discoveries provided by the international scientific community. Therefore, the source of this type of communication is represented by medical and scientific publications reporting data generated from basic science and clinical research. Health communications are targeted toward the general public, where the source is represented by health communicators and journalists. In health communications, information is the result of some kind of intra-language translation that allows transformation of the original medical language into a common language. Therefore, health communication derives from rewritings of a complex medical language that cannot always be modified and acquainted to serve the general public. The aim of the present work was to evaluate, in medical communications, the linguistic elements that represent the hard core for the general public. Thus, a qualitative evaluation was carried out on medical abstracts assessing medical terminology and compound phrases. The results of this investigation point out that these two linguistic traits of medical language are especially difficult for the general public due to their particular specialized nature.
\end{abstract}


Keywords: Medical communication, health communication, medical English, medical terms, compounds

\section{Introduction}

In the somewhat 'exceptional' conditions posed by the 2020 pandemic, medical knowledge transmission and spreading are crucial, especially because people need to be informed and reassured about the facts surrounding the disease and its spread (Cloitre and Shinn, 1985; Daniele, 2020). Indeed, the issue of knowledge and communication in medicine is certainly a serious one, and during this pandemic medical communications and health communications are taking place at different levels. In this context, the World Health Organization plays a key role, as well as the different health ministries and commissions in all countries, and doctors and journalists have also been ingaged (Gumperz, 1999). Health communication is represented by all journalistic genres, both oral (i.e. TV, the Internet, etc.) and written (WHO and government reports, newspapers, magazines, etc.), which have been widely used during this 2020 pandemic (Mocini, 2020; Sonke, 2020; Hsieh \& Kramer, 2021). Moreover, other genres include forms of professional communication like all expressions of doctor-patient interactions (Daniele, 2007; Langewitz, et al., 2021).

Health communications are targeted toward the general public, where the source is represented by health communicators and journalists. The information reported in health communications is the result of some kind of intra-language translation (plain language translation) that allows the transformation of the original medical language into a common language (Maurer, 2021). Health communication derives from rewritings of a complex medical language that cannot be always modified and acquainted to serve the general public. Indeed, language is a key element in any type of communication event; medical communications are written in a language shared by the scientific community, representing a specific linguistic community (Maurer, 2021).

Medical communication has many different features and facets, including a great variety of text and discourse genres. The progenitor of all these genres is certainly the research articles reporting scientific medical research and clinical trials in all the different branches of medicine and surgery (from human physiology to pediatrics, from cardiac to orthopedic surgery, from dermatology to psychiatry, etc.). Scientific medical research involves also epidemiological, diagnostic, pharmacological studies and treatment investigations (de Rothewelle, 2021).

Medical articles can be classified into two big categories: research articles and non-research articles, as they can be either the result of a scientific 
research process or not. These two written genres are extremely different in terms of both structure and function (Daniele, 2016).

The main difference between research articles and non-research articles is that the former report new, original data that have never been published previously; while the latter discuss already published scientific data using different forms of writings and publications. Research articles traditionally include the standard research genres known as full papers, rapid communications, short communications and abstracts. Furthermore, medical journals include a variety of non-research articles that are structurally similar to but functionally very different from research articles in terms of their purpose. The most important non-research articles are represented mainly by review papers, editorials, and letters to editors (Daniele, 2016).

Basic science or clinical research articles are all the result of medical researches, closely reflecting everything that occurs during scientific investigations. In medical academic journals, there are many types of research articles; the difference among them is functional rather than structural, and the various sections mirror exactly all the scientific events. Thus, although all research articles have the same structure as they all report the results of research procedures, actually, functionally they are different because their purpose is different (Daniele, 2016). Medical research articles have the wellknown IMRAD structure. This acronym derives from the main sections of research articles: introduction, materials and methods, or methods also called patients and methods (especially in clinical journals), results, discussion (Swales, 1990). However, research articles include also other important parts such as title, authors, affiliation, abstract, conclusion, keywords, acknowledgments, references, corresponding author, etc. (Daniele, 2016).

On the other hand, non-research articles have a variety of structures based on their particular function. As a matter of fact, they all lack the materials and methods section and the results section, as they do not derive from scientific researches. Sometimes, non-research articles may show a materials and methods section reporting the modalities used to attain all the information from the various data banks (i.e. PubMed-Medline, Google Scholar, etc.). Thus, non-research articles are principally made up of fewer sections that are mainly represented by: abstract, introduction, main text and conclusion (Daniele, 2016). It must be noted that non-research articles branch off into a multitude of genres, sub-genres and hypo-genres (Daniele, 2016).

There is a third category of medical papers that is identified in medical journals and it is called - case reports. In other words, papers of this kind have been qualified as borderline (Daniele, 2016), as they are neither research articles nor non-research articles. Case reports are writings mainly by clinicians who describe either new or uncommon diseases or known diseases that appear in peculiar novel forms that have never been seen before. The main 
purpose of case reports is to communicate to the scientific community that a new disease or new forms of known diseases have appeared. Furthermore, case reports allow researchers to interact with each other in order to share uncommon diagnostic procedures and treatments for peculiar clinical manifestations of rare and unknown diseases.

The aim of the present work was to evaluate, in the abstracts of medical publications, the linguistic elements that represent the hard core for the general public, like medical terminology and compound phrases. Thus, this investigation is targeted towards evaluating these two specific traits of medical language in light of their difficult understandability, which may sometimes invalidate the correct message delivered through health communications and reports.

\section{Methodology}

To address these issues a qualitative linguistic evaluation of medical abstracts was carried out. The abstracts were downloaded directly from the PubMed-Medline. To assess medical terms and compound phrases, over 100 medical abstracts were randomly selected using different search terms: Alzheimer's disease, breast cancer, cardiac disease, cervical cancer, colorectal cancer, diabetes, kidney disease, liver disease, low back pain, muscle dystrophy. These search terms were chosen randomly in the attempt to cover a large number of areas in medicine and surgery. The qualitative evaluation included assessment of medical terminology based on origin, like terms deriving from Latin and terms deriving from Greek. A second set of terms was selected based on meaning, like terms corresponding to an object and terms corresponding to a process. A third set of terms was selected according to lexical complexity, and a number of complex medical compound terms were appraised. Finally, possible synonyms with standard language were taken into consideration. Besides terminology, syntax was also assessed and particularly compound phrases. The qualitative evaluation of the abstracts was performed directly by the author of the present paper. All abstracts were examined by introducing search terms that could enable finding of particular medical terms, roots, prefixes and suffixes.

\section{Results}

\section{Understanding Medical Terms}

Medical language is enormously full of technical terms, which are words belonging in a particular register used by a specific linguistic community. Medical terms can be classified in different ways. 
1. In medical English, terms can be classified based on their lexical/syntactic origin.

A number of technical terms derive from Latin:

$\begin{array}{ll}\text { Alveolus } & \text { alveoli } \\ \text { Embolus } & \text { emboli } \\ \text { Focus } & \text { foci } \\ \text { Bacterium } & \text { bacteria } \\ \text { Lamina } & \text { laminae }\end{array}$

Some terms derive from Greek:

$\begin{array}{ll}\text { Apex } & \text { apices } \\ \text { Lysis } & \text { lyses } \\ \text { Necrosis } & \text { necroses } \\ \text { Diagnosis } & \text { diagnoses } \\ \text { Prognosis } & \text { prognoses }\end{array}$

2. Medical terms can be classified based on their number, and some are only plural without a singular:

Lymphatics

Some are only singular:

Information

Equipment

Some singular medical terms end with 's':

Genetics

Diabetes

Diuretics

Digitalis

Kinetics

Some medical terms are equal in their singular and plural forms:

This series presents three additional cases.

Five series have used twice-daily radiotherapy.

Some medical terms have both a different plural and meaning in medical and standard language:

Appendix appendixes appendices

Index indexes indices 
3. Some medical terms can derive from adding 'ing':

Monitoring

Screening

4. Medical terms can be classified based on their meaning. Words that correspond to an object or a substance:

Acetylcholinesterase

Neurotransmitter

Amyloid-beta

Neuron

Sarcolemma

Words that correspond to a process:

Pathogenesis

Aetiopathogenesis

Histology

Meiosis

Apoptosis

Some change their meaning:

Analysis of group means indicated many differences.

Biochemical assays are the most reliable means for detecting myocardial injury.

Some change their meaning in standard and medical language:

Soldiers stood sentinel with their muskets.

A sentinel surveillance program for influenza.

Investigations involved analyzing samples of handwriting.

Blood samples were collected from all patients.

5. Few terms have a synonym in standard and medical language:

Anamnesis history

Cutaneous skin

Etiology cause

Hemorrhage bleeding

Intestine gut

Stenosis stricture

Thorax chest

Compound words

Medical writings are rich in compound words. Word compounding is the formation of medical terms starting from more than one lexical item, and 
it is used for the purposes of concision (Crespo, 2011). All compound words begin with a root, which in medicine originates predominantly from Latin or Greek. When creating compound words different mechanisms can be involved.

1. A prefix or a suffix is added to the root.

The root -cardio- meaning heart:

Brady-cardia, tachy-cardia

Cardi-ac, cardio-logist

Cardio-vascular, cardio-protective

The root -parathyro- meaning parathyroid:

Hyper-parathyro-idism, hypo-parathyro-idism

Parathyro-iditis

2. Only a prefix is added to the root:

Neuro-transmitter

Extra-ventricular

Hemi-paresis

Vaso-protective

3. Only a suffix is added to the root:

Endotheli-al

Nerv-ous

Ischem-ic

Carri-er

4. Another way of constructing compound words is through the combination of two or more roots:

Sensori-neural

Acetyl-cholin-esterase

Angio-pathy

Anti-thrombot-ic

5. Compound words can be generated through the combination of two or more words joined by a hyphen:

Anthracycline- and taxane-containing chemotherapeutic regimens

Hypertension-induced left ventricular hypertrophy

Stress-related cardiac conditions

Blood-brain barrier

Understanding Compound Phrases

One of the principal features of medical writings is their lexical density. Lexical density is produced when the number of function words is 
significantly less represented than that of lexical words. Both high lexical density and the alteration of the relation between function words and lexical words are obtained through pre-modification and compounding (Daniele, 2019). A particular modality of generating compound phrases is called nominalization. In this process of phrase formation, an adjective or a verb is used as a noun. Functional grammar refers to nominalization as a transformation of an underlying clause into a noun phrase (Halliday, 1989). Nominalization is closely related to pre-modification and compounding. Premodification and compounding have no precise rules for their development, and many ways can be followed to create pre-modified phrases, also known as compound phrases. Pre-modification is the creation of strings of words ending with a head noun. Countless words called modifiers actually 'modify' the head noun that is located at the very end of the string of words. The premodification process involves the removal of all function words, including verbs (Duam and $\mathrm{Gu}, 2010$ ). In this way, the whole writing acquires more fluidity and is, therefore, more accessible. Nominalization together with premodification and compounding all tend to reduce the number of function words and make the text more 'dense' with lexical words (Zorita and Sandoval, 2016). Actually, the compounding process just described yields compound phrases, which differently from sentences, are syntactic structures constituted by strings of at least two nouns lacking both function words and verbs. Indeed, compound phrases contain only adjectives and nouns, even though these latter can act as adjectives. In other words, in compounds, nouns are always invariable, meaning that they cannot be plural, except for the head noun. Indeed, when the number of the noun inside the string of words cannot be deduced, the formation of compound phrases should not be undertaken, so to avoid confusion. The specific features of compound sequences, which can contain two or more words harbor an enormous interpretation complexity. Thus, intra-language translation and decoding of these specific peculiar syntactic structures pose particular problems. Repositioning of the different words in the right place in the string of words, their understanding, as well as having to reintroduce the function words and sometimes the verbs are particularly arduous actions.

1. Noun-noun:

Transcription factor

Cell injury

Nephrectomy patients

Creatinine measurements

2. Noun-adjective-noun:

Heart muscular apparatus

Gene sarcolemmal integrity 
3. Adjective-noun-noun:

Endothelial cell function

Diabetic kidney disease

4. Adjective-adjective-noun:

Normal vascular integrity

Glomerular endothelial cells

5. Adjective-adjective - noun-noun:

Endothelial nitric oxide synthase

Subclinical epileptic seizure activity

6. Various combinations:

Node-positive breast cancer

Hormone receptor-positive cancer

Breast cancer treatment options

Sentinel lymph node biopsy

Adjuvant systemic therapy

Experimental animal model studies

Amyloid precursor protein processing

National cervical cancer screening program

Proline-to-leucine (P448L) mutation

Increased endothelial nitric oxide synthase expression

The different combinations are potentially infinite because the abundance and availability of lexical words in medicine are exceptionally large. Different ways of analyzing compounds can be assumed, and understanding compound phrases is a kind of intra-linguistic translation process. Therefore, some of the mechanisms used for inter-linguistic translations can be well applied to understanding these complex medical phrases (Rackow, 1992). Another way of understanding compound phrases is through compound splitting, which is involved with separating the words, translating every single word and then re-sensing them in the target language (Koehn and Knight, 2003). Finally, Bylan and Chaterjee (2015) have worked with machine translation of compound phrases, establishing a three-step scheme. The translation process starts with the identification of a noun compound phrase. Then, a semantic relation is determined among the words in the compound phrase. Lastly, a translation pattern is selected; many translating patterns have been created for 2-word, 3-word, 4-word noun compound phrases. 


\section{Conclusion}

The world of medical communication is a fascinating one, as it possesses enormous potentials that can change humans' lives in many ways. Most of the medical information is written in English, which has become the Lingua Franca internationally utilized for medical and scientific publications. English has replaced the important communicative role played in the past by languages like Latin and Greek. Mostly, medical language has a communicative function, and it may be classified based on the target it is addressed to. It can also be defined as a variation of standard language characterized by a particular lexical apparatus and a precise set of syntactic and grammatical rules and norms. In terms of mode, medical language can be either written or oral based on its use, and both these forms have a technical constitution, as they are addressed to a specific scientific community. In this way, the specific scientific community becomes a specific linguistic community, as it shares a common language. Medical language expresses also the formal nature of all the specialized, technical languages that are necessary for effective academic interactions. Indeed, medical language can be distinguished in the language of medical occupation (in academic journals), which represents the parent from which all other forms of specialized discourses derive, and primarily the language of medical education (textbooks) and medical technical language (manuals). Medical language is commonly referred to as Medical English, which has actually been named in many different ways: English for Occupational Purposes (EOP), English for Academic Purposes (EAP), and English for Special/Specific Purposes (ESP).

In conceptualizing medical language, it must be pointed out that as a special language targeted to a specific linguistic community, it is endowed with a myriad of unwritten rules and norms that are difficult for 'outsiders' to understand and apply. Furthermore, some of the most difficult traits of medical language for non-professionals are certainly terminology and compound phrases, which were addressed in this research.

In the over 100 medical abstracts that were randomly chosen from the publications appearing in the PubMed-Medline, the qualitative evaluation showed that an enormous variety of technical terms are present in medical abstracts, which can be classified in different ways. Medical terms are extremely rich in all types of roots to which prefixes and suffixes can be added in various ways; the roots can be also joined together in many aspects to create new words. New terms can be the result of combinations of two or more words linked together by hyphens, which generate complex novel challenging terms. Overall, medical terms can be categorized according to either their lexical/syntactic origin or the absence/presence of singular and plural forms. Another way of viewing medical terms is through investigating into their meaning, as they correspond to specific objects and activities that belong 
uniquely to the scientific and medical world. Only a negligible number of medical terms have a synonym in standard language, most of them are unique terms that cannot be replaced, and this is the very difficulty of medical terms.

All the mechanisms aimed at generating new terms lead to the high lexical density that characterizes medical language; lexical density is also the result of the creation of strings of words known as compounds. Compound phrases are the second linguistic item that was studied in this investigation. The process of phrase formation using compounding and nominalization creates a language that is exceedingly difficult to be understood by the general public, as it contains too many unwritten rules. Compound phrases represent a real challenge for non-specialists as their decoding requires not only a good knowledge of the grammatical structures they possess but also of the meaning of every single word that appears in the string. Indeed, the present investigation evidenced that the overuse of these linguistic structures together with the resulting lexical density are concrete complications for health journalists, health communicators and the general public, where the only loophole is attempting to make sense out of each string of words. Two processes have been described that help codify compound phrases - compound splitting and the three-step scheme.

In health communications, specialized journalists and health communicators, in general, need to understand and master the peculiarities of medical language. In this way, they can be able to transform the medical language into the language of medical journalism - popular medicine and to convey correct messages to non-specialized audience. Specialized journalists and health communicators should be acquainted with the terminology that pervades medical articles and especially with the terms related to every single branch of medicine, which is indeed diversified and always new. In this context, specialized dictionaries could serve this purpose; however, these specialized dictionaries are not always equipped with all the apparatuses that enable translation of complex meanings into simpler words and forms. With medical terminology, the intra-linguistic translation of the intriguing concepts and mechanisms should be carried out not so much trying to substitute the words with synonyms or correspondents, but with in-depth explanations, because as previously stated, only a limited number of terms actually do possess a synonym in standard language.

Much work and research are necessary to further investigate into these themes that were only mentioned in the present paper to gain more insight into the remarkable world of medical language. Indeed, to date, medical language still remains a special language for a specific community; its transformation into standard language requires a number of strategies that are not always successful in delivering the message, and its understanding is not always possible. 


\section{References:}

1. Balyan R. and Chatterjee N. (2015). Translating noun compounds using semantic relations. Computer Speech \& Language, XXXII, 1, pp. 91-108 - Doi.org/10.1016/j.csl.2014.09.007.

2. Cloître M. and Shinn T. (1985). "Expository Practice: Social, Cognitive and Epistemological Linkages". In: Shinn T. and Whitley R. (Eds.), Expository Science: Forms and Functions of Popularization. Dordrecht, Reidel, pp. 31-60.

3. Crespo B. (2011). Rosewater, wheel of fortune: Compounding and lexicalisation in seventeenth-century scientific texts. Nordic Journal of English Studies, 10 (1), pp.135-154.

DOI: http://doi.org/10.35360/njes.245

4. Daniele F. (2007). "Bridges to Language Barriers". In: Cityscapes the Islands of the Self, Proceedings of the Associazione Italiana di Anglistica, Cagliari, pp. 173-184.

5. Daniele F. (2016). "A Dynamic Classification of Medical Genres". In: Daniele F. \& Garzone G. Communicating Medicine, Popularizing Medicine - Lingua Traduzione Didattica. Milano, FrancoAngeli.

6. Daniele, F. (2019). Performance of an automatic translator in translating medical abstracts. Heliyon, 5, pp. 1-6.

https://doi.org/10.1016/j.heliyon.2019.e02687

7. Daniele, F. (2020). "Preface: The Language of Pandemics". In: Daniele F. The Language of Pandemics. European Scientific Journal, ESJ, 16(38), 1. https://doi.org/10.19044/esj.2020.v16n37p1

8. de Rothewelle J. (2021). "Medical Communication and SARS-CoV-2: Novel Approaches to Global Health Crises Communication as Called for by the W.H.O." In: Outpatient Care.

DOI:10.5772/intechopen.95943

9. Duan P. and Gu W. (2010). Noun-string Pre-modification in Technical English Writing [J]. Chinese Science \& Technology Translators. Google Scholar.

10. Gumperz J. (1999). "On Interactional Sociolinguistics Method" in Sarangi S. and Roberts C. (Eds.), Talk, Work and Institutional Order: Discourse in Medical Mediation and Management Settings. Berlin, Mouton de Gruyter, pp. 453-471.

11. Halliday M.A.K. (1989). Some grammatical problems in scientific English. Review of Applied Linguistics, Supplement Series 5, 6, pp. 13-37. 
12. Hsieh E. and Kramer E.M. (2021). Rethinking culture in health communication: Social interactions as intercultural encounters. Wiley Blackwell.

13. Koehn P. and Knight K. (2003). "Empirical methods for compound splitting". Proceedings of the European Chapter of the Association for Computational Linguistics (Budapest, Hungary).

14. Langerwitz W. (2021). Doctor-patient communication during the Corona crisis - web-based interactions and structured feedback from standardized patients at the University of Basel and the LMU Munich. Journal for Medical Education, 38(4). doi: 10.3205/zma001477

15. Maurer M., et al., (2021). Lessons Learned from Developing Plain Language Summaries of Research Studies. Health Literacy Research and Practice, Vol. 5, No. 2, pp. 155-161. https://doi.org/10.3928/24748307-20210524-01

16. Mocini R. (2020). "The Voice of Authority Vis-À-Vis the Covid Pandemic". In: Daniele F. The Language of Pandemics. European Scientific Journal, ESJ, 16(38), 1. doi: 10.19044/esj.2020.v16s2p1.

17. Rackow U., et al., (1992). "Automatic translation of noun compounds". Proceedings of the 15th International Conference on Computational Linguistics, I.

18. Sonke J., et al. (2020). Health Communication and the Arts in the United States: A Scoping Review, American Journal of Health Promotion, 35(1), 106-115. https://doi.org/10.1177/0890117120931710.

19. Swales J.M. (1990). Genre Analysis - English in academic and research settings. New York, Cambridge University Press.

20. Zorita C.H. and Sandoval A.M. (2016). "Sentence length and NP complexity of general and medical written academic and media texts. An analysis using a trained syntactic parser". - researchgate.net. 